\title{
BMJ Open Association between family history of diabetes and clusters of adherence to healthy behaviors: cross-sectional results from the Health Examinees-Gem (HEXA-G) study
}

\author{
Jaesung Choi, ${ }^{1}$ Ji-Yeob Choi, ${ }^{1,2,3}$ Sang-Ah Lee, ${ }^{4}$ Kyoung-Mu Lee, ${ }^{5}$ Aesun Shin, ${ }^{2,3}$ \\ Juhwan Oh, ${ }^{6}$ JooYong Park, ${ }^{1}$ Minkyo Song, ${ }^{2,7}$ Jae Jeong Yang, ${ }^{2}$ Jong-koo Lee, ${ }^{6,8}$ \\ Daehee Kang ${ }^{1,3,2,9}$
}

To cite: Choi J, Choi J-Y, Lee S-A, et al. Association between family history of diabetes and clusters of adherence to healthy behaviors: cross-sectional results from the Health Examinees-Gem (HEXA-G) study. BMJ Open 2019;9:e025477. doi:10.1136/ bmjopen-2018-025477

- Prepublication history and additional material for this paper are available online. To view these files, please visit the journal online (http://dx.doi. org/10.1136/bmjopen-2018025477).

Received 17 July 2018 Revised 22 March 2019 Accepted 15 May 2019

Check for updates

(C) Author(s) (or their employer(s)) 2019. Re-use permitted under CC BY-NC. No commercial re-use. See rights and permissions. Published by BMJ.

For numbered affiliations see end of article.

Correspondence to

Dr. Ji-Yeob Choi;

jiyeob.choi@gmail.com

\section{ABSTRACT}

Objectives This study evaluated whether individuals with affected family member adhered to healthy behaviours.

Design and setting This was a cross-sectional study of participants selected from health examinees who underwent the national health check-up programme of Korea in 39 centres between 2004 and 2013.

Participants The baseline data of 128520 participants enrolled in the Health Examinees-Gem study were used for analysis.

Main outcomes and measures Associations of family history of diabetes with adherence to regular exercise, healthy diet and body composition, and clusters of healthy behaviours were evaluated while adjusting for potential confounders selected by a directed acyclic graph.

Results Participants with a family history of diabetes were more likely to adhere to a regular exercise regimen $(\mathrm{OR}=1.12,95 \% \mathrm{Cl} 1.06$ to 1.18 for men and $\mathrm{OR}=1.10$, $95 \% \mathrm{Cl} 1.07$ to 1.14 for women) and healthy diet $(\mathrm{OR}=1.06,95 \% \mathrm{Cl} 1.01$ to 1.12 for men and $\mathrm{OR}=1.06$, $95 \% \mathrm{Cl} 1.01$ to 1.12 for women) but were less likely to have a normal body composition $(\mathrm{OR}=0.83,95 \% \mathrm{Cl} 0.78$ to 0.87 for men and $\mathrm{OR}=0.83,95 \% \mathrm{Cl} 0.80$ to 0.86 for women). These associations were strengthened when the affected family members were siblings, the number of affected members was increased or the age at diagnosis of the affected member was younger than 50 years. In men and women, having a normal body composition is important in determining the cluster of behaviours, and those with a family history of diabetes were less likely to adhere to the normal body composition cluster.

Conclusions The group with high risk of diabetes showed healthy behaviors, but they did not have a normal body composition. Policies and campaigns targeting integrated health behaviors will be needed to reduce the burden of diseases and improve public health.

\section{INTRODUCTION}

Diabetes is a major cause of death and disability worldwide. ${ }^{1}$ The prevalence of and mortality from diabetes has increased more
Strengths and limitations of this study

- This is the first study to comprehensively evaluate the association of the relationship type, number and age at diagnosis of affected family members with adherence to behavioral factors.

- A large sample size, including 128520 middle-aged men and women, allowed testing of the association with sufficient statistical power.

- As the information on the family history of diabetes and lifestyle behaviors was collected by self-reported questionnaire, the estimates of the associations could have been biased.

than any other non-communicable disease during the last decade. ${ }^{2}$ Worsened quality of life from diabetes, assessed by years lost due to disability, increased more than the other top 10 non-communicable diseases between 1990 and $2015 .{ }^{3}$ The prevalence of diabetes in Korea is $13.7 \%$ and is expected to increase, as approximately one-quarter of the Korean population is currently in a prediabetic state. ${ }^{4}$

In the lastfew decades, it has been established that diabetes can run in families, and people with a family history of diabetes are at a higher risk for developing diabetes. ${ }^{5}{ }^{6}$ Behavioural change is the best-known modifiable risk factor, and healthy behaviours are known to be associated with a $40 \%-80 \%$ reduction in the risk of developing diabetes. ${ }^{7-11}$ Based on this evidence, the American Diabetes Association, the National Institute of Diabetes and Digestive and Kidney Disease and the Korea Centers for Disease Control and Prevention established guidelines that suggest adopting healthy behaviours, such as regular exercise, a healthy diet and having a normal body composition, to prevent diabetes. ${ }^{12-14}$ Thus, 
it is important to monitor whether high-risk people are following preventive guidelines.

It has been reported that family history can cause perception of disease risk, and those who have a family history were more likely to have greater perceived risk for diabetes. ${ }^{1516}$ However, there were disagreements on whether or not family history has a practical impact on behaviours by inducing perception of diseases. ${ }^{17}{ }^{18} \mathrm{In}$ previous studies, which evaluated the association between a family history of diabetes and behavioural factors, there were inconsistent results in physical activity, ${ }^{19-26}$ healthy diet $^{2022} 232526$ and body composition. ${ }^{19-26}$ However, in these studies, characteristics of family history such as the relationship type and number of family members affected and age at diagnosis of affected family members were not considered, although these characteristics could affect the perception of a person's vulnerability to disease ${ }^{27}$ and should, therefore, be of interest when studying the effect of family history.

Behaviours typically cooccur or cluster together in the real world. ${ }^{28}$ Patterns of multiple behaviours are known to have a synergistic health effect, that is, greater than the sum of the effects of each behaviour. ${ }^{29}$ The application of an intervention targeting multiple behaviours in those with unhealthy patterns was more effective than an intervention that targeted a single factor. ${ }^{30}$ Thus, it is important to consider multiple factors when assessing the effect of health behaviours on public health. However, patterns of healthy behaviours in association with family history have not been evaluated.

The main purpose of the present study was to assess whether the associations between family history of diabetes and healthy behaviours, such as regular exercise, a healthy diet and having a normal body composition, ${ }^{12-14}$ were affected by the relationship type, number and age at diagnosis of affected family members. In addition, we assessed how family history-related characteristics were associated with clusters of these healthy behaviours.

\section{METHODS}

\section{Study population}

This cross-sectional study was conducted among the baseline participants of the Health Examinees-Gem (HEXA-G) study derived from the Health Examinees study, a component of the Korean Genome and Epidemiology Study (KoGES_HEXA). KoGES_HEXA is a largescale genomic cohort study that recruited participants aged 40-69 between 2004 and 2013. The rationale, design and baseline characteristics of the KoGES_HEXA cohort study have been described elsewhere. ${ }^{31}{ }^{32}$ During recruitment, a consent form was voluntarily signed by all participants before entering the study. In the HEXA-G study, 139344 participants composed of 46977 men (33.7\%) and $92367(66.3 \%)$ women were included at baseline after excluding participants recruited at 21 centres that had only operated in a pilot study, that had different processes for quality control and biospecimen collection, had been participating for <2years or were no longer participating in the cohort. Additionally, 10824 participants were excluded due to the following criteria: missing information regarding family history of diabetes, regular exercise, food frequency questionnaire and body composition, such as body mass index (BMI) or waist-hip ratio (WHR); participants who have an implausible caloric intake of $<800$ or $\geq 4000 \mathrm{kcal} /$ day in men and $<500$ or $\geq 3500 \mathrm{kcal} /$ day in women; and participants who have only affected children rather than affected parents or siblings to rule out the influence of type 1 diabetes, which mostly develops during childhood (figure 1). Finally, 128520

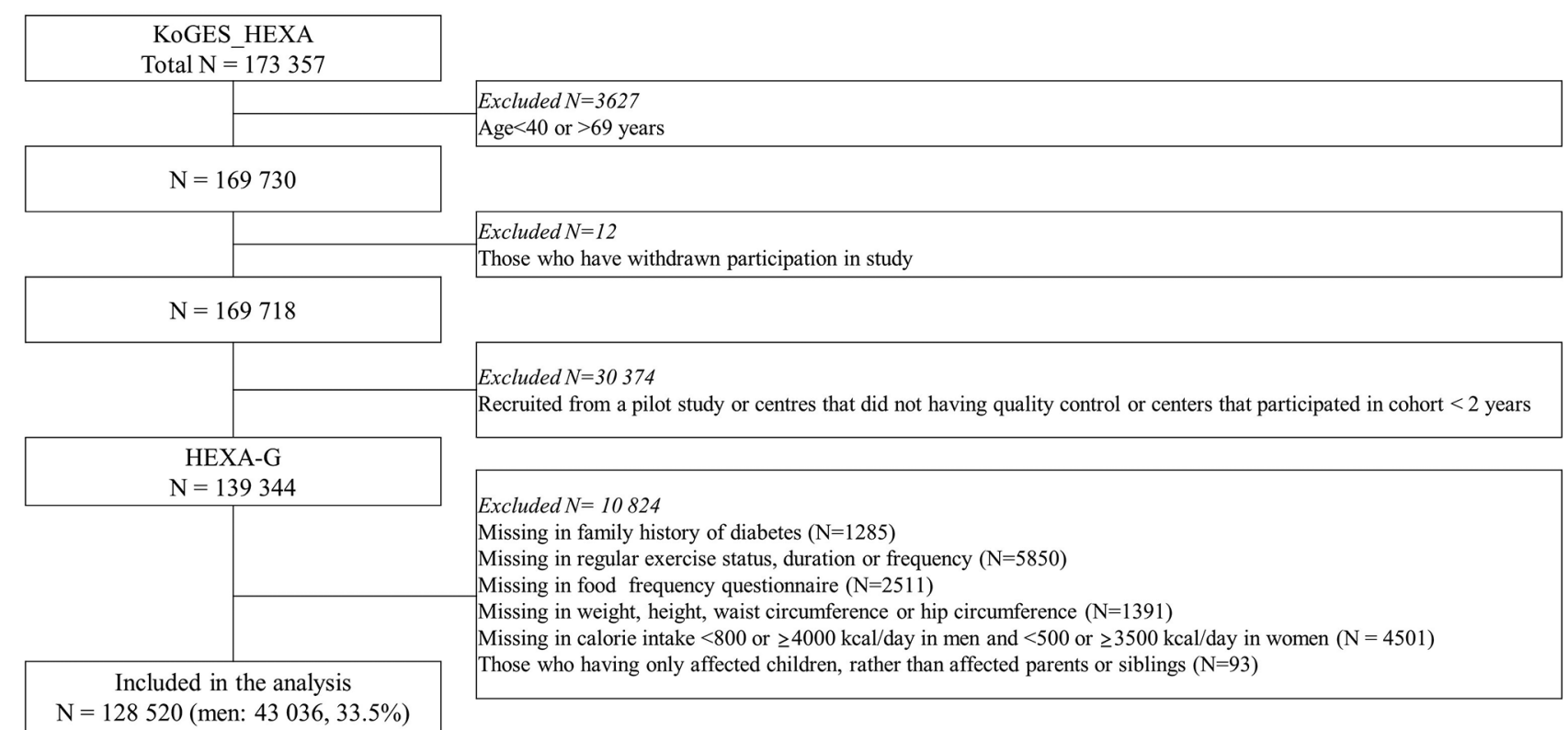

Figure 1 Inclusion and exclusion criteria of the study population. HEXA-G, Health Examinees-Gem; KoGES_HEXA, Korean Genome and Epidemiology Study. 
participants including 43036 men $(33.5 \%)$ and 85484 women $(66.4 \%)$ were included in the analysis. Among these participants, $18.5 \%$ (95\% CI 18.3 to 18.7 ) had a family history of diabetes.

\section{Family history}

A family history of diabetes was assessed by asking whether participants had a first-degree family member diagnosed with diabetes. In those with a family history of diabetes, information on the relationship type of affected family member, such as parents, siblings or offspring, was also collected for each affected member. Participants were then classified into three groups: no family history, any affected parents and any affected siblings. Participants with affected parents or siblings were not exclusively classified. For example, the category of having any affected parents included those who had affected parents with or without affected siblings. The total number of affected family members was tallied from the number of responses for each relationship type of affected family member, and participants were then classified into three groups: no family history, one affected family member and two or more affected family members. The age at diagnosis of each affected member was collected. Since type 2 diabetes diagnosed at a younger age has a more aggressive disease phenotype leading to a worse prognosis ${ }^{33}$ as well as higher risk of diabetes in unaffected family members, ${ }^{22}$ a younger age of the affected family member may induce a greater impact on family members' perception of the condition. Fifty years was chosen as the cut-off because a higher risk of diabetes was reported when there were family members diagnosed before the age of 50 years. ${ }^{22}$ Participants were then classified into three groups: no family history, age at diagnosis of the affected family member younger than 50 and 50 years or older. Among those who have two or more affected family members, the youngest age at diagnosis of an affected member was selected.

\section{Adherence to healthy behaviours}

Self-reported behavioural factors, such as regular exercise, a healthy diet and measured body composition, were included in the analysis. Participants were asked if they performed regular exercise (enough to sweat), the duration of each exercise session and were asked about the frequency of exercise per week. The duration of regular exercise per week was calculated by multiplying the duration of each session by the frequency per week. Participants doing $>150 \mathrm{~min} /$ week were categorised into the adherence group. ${ }^{34}$ Healthy diet was assessed by the diet quality index for Koreans (DQI-K) developed based on the original diet quality index (DQI) ${ }^{35}$ The DQI-K scores were calculated by scoring and summing the daily intake patterns of eight components, including protein, fat, saturated fat, cholesterol, whole grains, fruit, vegetables and sodium, which were categories from the original DQI but replaced carbohydrates with whole grains. The DQI-K scores ranged from 0 to 9, and lower DQI-K scores indicated a higher-quality diet. Since DQI-K was reported to have a linear association with mortality ${ }^{35}$ and there is no standard cut-off, participants with a DQI-K score at the median or lower (DQI-K scores ranged 0-3) were defined as the adherence group for statistical stability. BMI and WHR were used as indicators of obesity-related body composition. BMI was calculated as measured weight in kilograms divided by measured height in metres squared, and WHR was calculated as measured waist circumference divided by measured hip circumference. The anthropometric indicators were accurate to $0.1 \mathrm{~kg}$ for weight and $0.1 \mathrm{~cm}$ for height, waist circumference and hip circumference. Participants whose BMI was $<25.0$ and WHR $<0.90$ in men and 0.85 in women were defined as the adherence group. ${ }^{36} 37$

\section{Potential covariates}

Demographic factors, family history of cardiovascular diseases (CVD) and cancer, and prior diagnosis of diabetes, CVD and cancer were selected a priori as potential covariates. Demographic factors included age (40-49, 50-59 and 60-69 years), educational level ( $\leq$ middle school, high school graduate and $\geq$ college), monthly income in Korean currency $(<2.0$ million won, 2.0-3.9 million won and $\geq 4.0$ million won ( 1 million won is $\sim$ US $\$ 1000$ ), and current occupational status (office, manual and unemployed or housewife). Participants were categorised into having a family history of other diseases, such as CVD and cancer, if they reported an affected family member with these diseases. Prior diagnosis of diabetes, CVD or cancer was defined as participants who have been diagnosed with these diseases by a doctor at a hospital or clinic.

\section{Statistical analysis}

All analyses were performed separately by sex. To identify the minimum set of confounders, a directed acyclic graph (DAG) was drawn based on an a priori assumption between potential covariates, exposure and outcome variables (online supplementary figure 1). The DAG was drawn by using DAGitty web-based software (http:// www.dagitty.net).$^{38}$ Because age was the only confounder between each covariate and adherence to each healthy behaviour in the DAG ((A) of online supplementary figure 1), an age-adjusted logistic regression model was used to calculate the OR and $95 \%$ CI for the associations between them. In the analysis between family history and adherence to each healthy behaviour, the variables including age, education, occupation, income and family history of CVD and cancer were identified as confounders by DAG ((B) of online supplementary figure 1$)$. To avoid multicollinearity, the variables with variance inflation factors $>10$ were excluded in the binary logistic regression model. Finally, all confounders, except for family history of cancer, were included in the model. In the analysis of the relationship types of family history and each behaviour, associations of affected parents and affected siblings with adherence to each healthy behaviour were separately conducted because participants were not exclusively classified. For example, the OR and $95 \%$ CI for the affected 

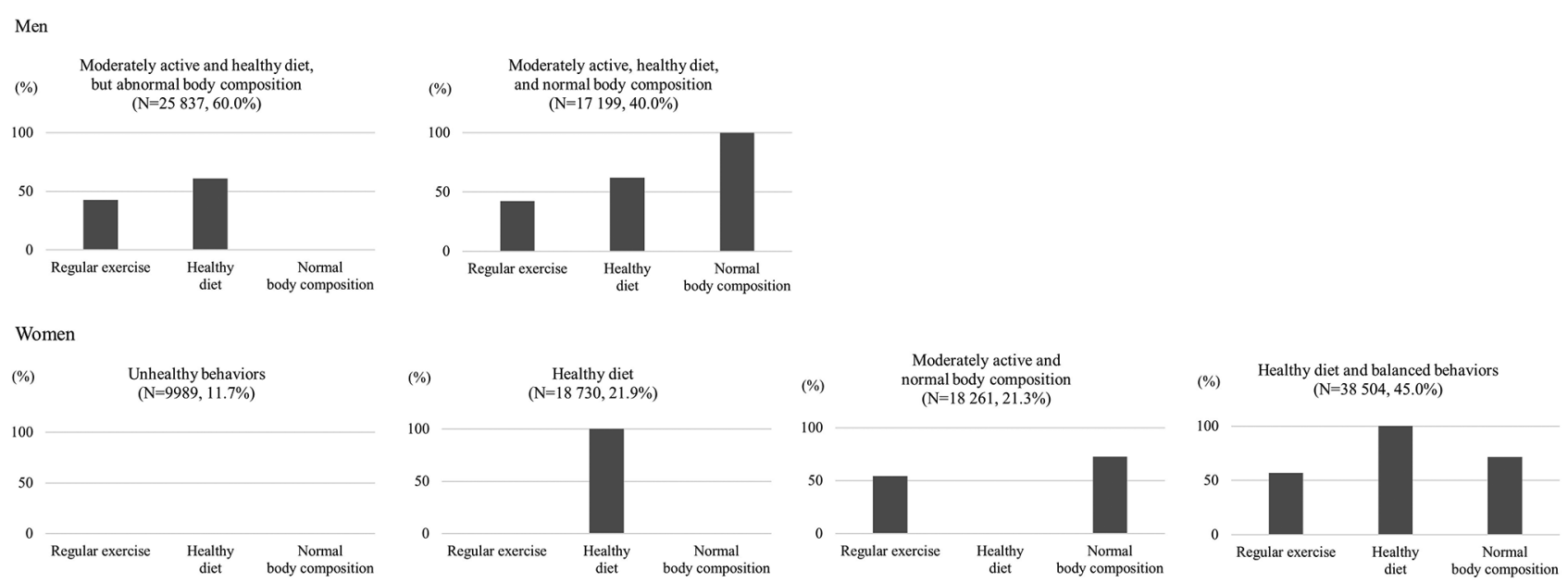

Figure 2 Clusters of healthy behaviours in men $(n=43036)$ and women $(n=85484)$.

parents were evaluated in comparison to participants with no family history while those with affected members other than parents were included in the model, which were not shown. In the binary logistic regression, we performed a trend test for the number of affected family members. Interactions between family history of diabetes and sex with adherence to behaviours were assessed by computing $\mathrm{p}$ values from likelihood ratio tests by comparing models with and without the interaction terms. The goodness of model fit was evaluated by the Hosmer-Lemeshow goodness of fit test, and most adjusted models between family history of diabetes and each healthy behaviour satisfied the goodness of fit $(p>0.05)$.

Cluster analysis was performed to find the pattern of healthy behaviours according to sex. This method is useful for identifying co-occurring healthy behaviours by subgrouping participants with similar patterns of behaviours. We used the PROC HPCLUS procedure in SAS software, which performed a k-means clustering algorithm and determined the most suitable number of clusters in a set of data by comparing the sum of squares error between input data and expectation. ${ }^{39}$ Cluster analysis revealed two clusters in men and four clusters in women (figure 2). The characteristics of each cluster in men and women were defined based on the proportion of participants adhering to each healthy behaviour in each cluster (y-axis of figure 2). The associations of family history and each cluster were evaluated by binary (for men) or multinomial logistic regression (for women) with the same model described above, and the unhealthiest behaviour cluster was used as a reference in each sex.

We also conducted sensitivity analysis in only participants without a prior diagnosis of diabetes and without a prior diagnosis of diabetes, CVD and cancer. All statistical analyses were performed with SAS V.9.4. All $\mathrm{p}$ values were two-sided, and a $\mathrm{p}$ value of $<0.05$ was considered statistically significant.

\section{Patient and public involvement}

No patients or public were involved in setting the research question, study design and interpretation of this study.
However, patient data and resource access have been approved by the governance office. The results will not be disseminated to study participants.

\section{RESULTS}

Among the eligible 128520 participants (43036 men and 85484 women), the mean age was 53.5 (8.4) years for men and 52.3 (7.8) years for women. The prevalence of a family history of diabetes was $16.3 \%$ (95\% CI 15.9 to 16.6) for men and $19.7 \%$ (95\% CI 19.4 to 19.9) for women (online supplementary table 1). Table 1 presents associations between potential covariates and adherence to each healthy behaviour. Among both men and women, older participants more frequently reported an adherence to regular exercise and healthy diet but not to having a normal body composition. Adherence to regular exercise was positively associated with high levels of education and income, office jobs or unemployment, family history of CVD and cancer, and prior diagnosis of diabetes and cancer. There were similar associations of healthy diet with potential covariates, except for education in women and incomes in both men and women. Adherence to having a normal body composition was positively associated with higher education, unemployment and prior diagnosis of cancer and was negatively associated with prior diagnosis of diabetes and CVD. The associations between potential covariates and a family history of diabetes are also presented in online supplementary table 2.

Table 2 shows the associations of a family history of diabetes with adherence to each healthy behaviour. Participants with a family history of diabetes were more likely to participate in regular exercise $(\mathrm{OR}=1.12,95 \% \mathrm{CI}$ 1.06 to 1.18 for men and $\mathrm{OR}=1.10,95 \%$ CI 1.07 to 1.14 for women) and practice a healthy diet (OR=1.06, 95\% CI 1.01 to 1.12 for men and OR=1.05, 95\% CI 1.02 to 1.09 for women). Those participants having a family history of diabetes were less likely to adhere to having a normal body composition ( $\mathrm{OR}=0.83,95 \%$ CI 0.78 to 0.87 for men and $\mathrm{OR}=0.83,95 \%$ CI 0.80 to 0.86 for women). Most of 


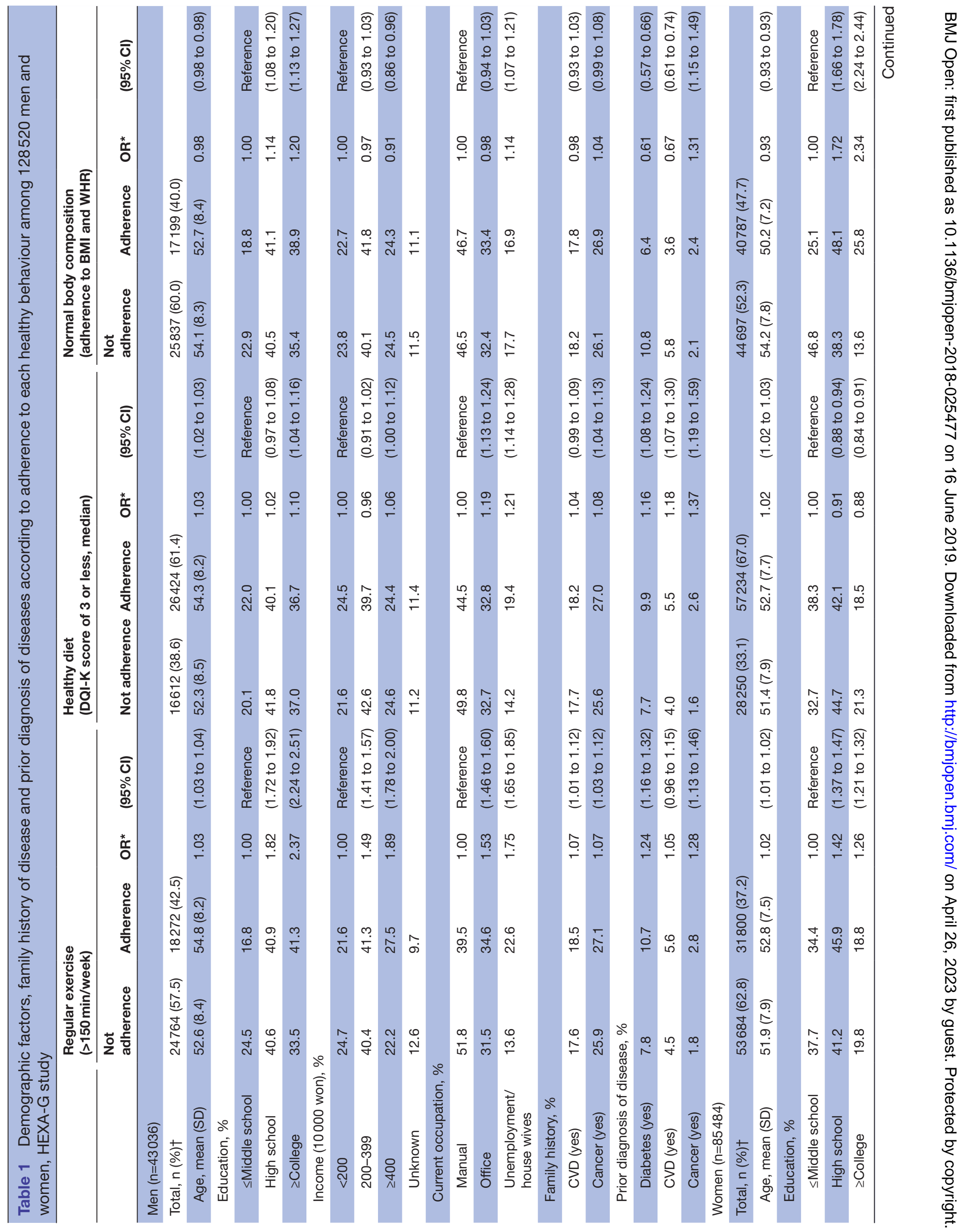




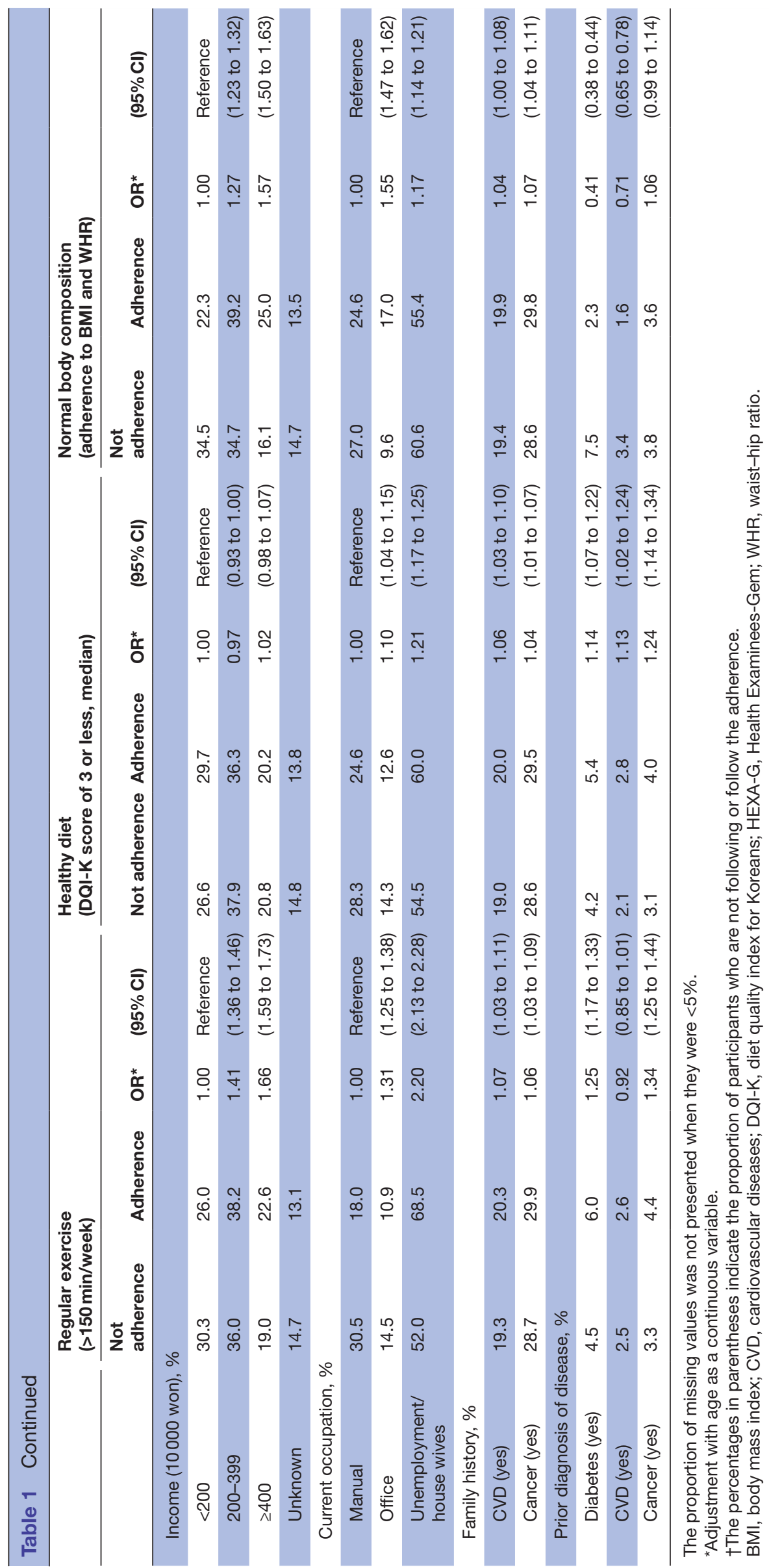




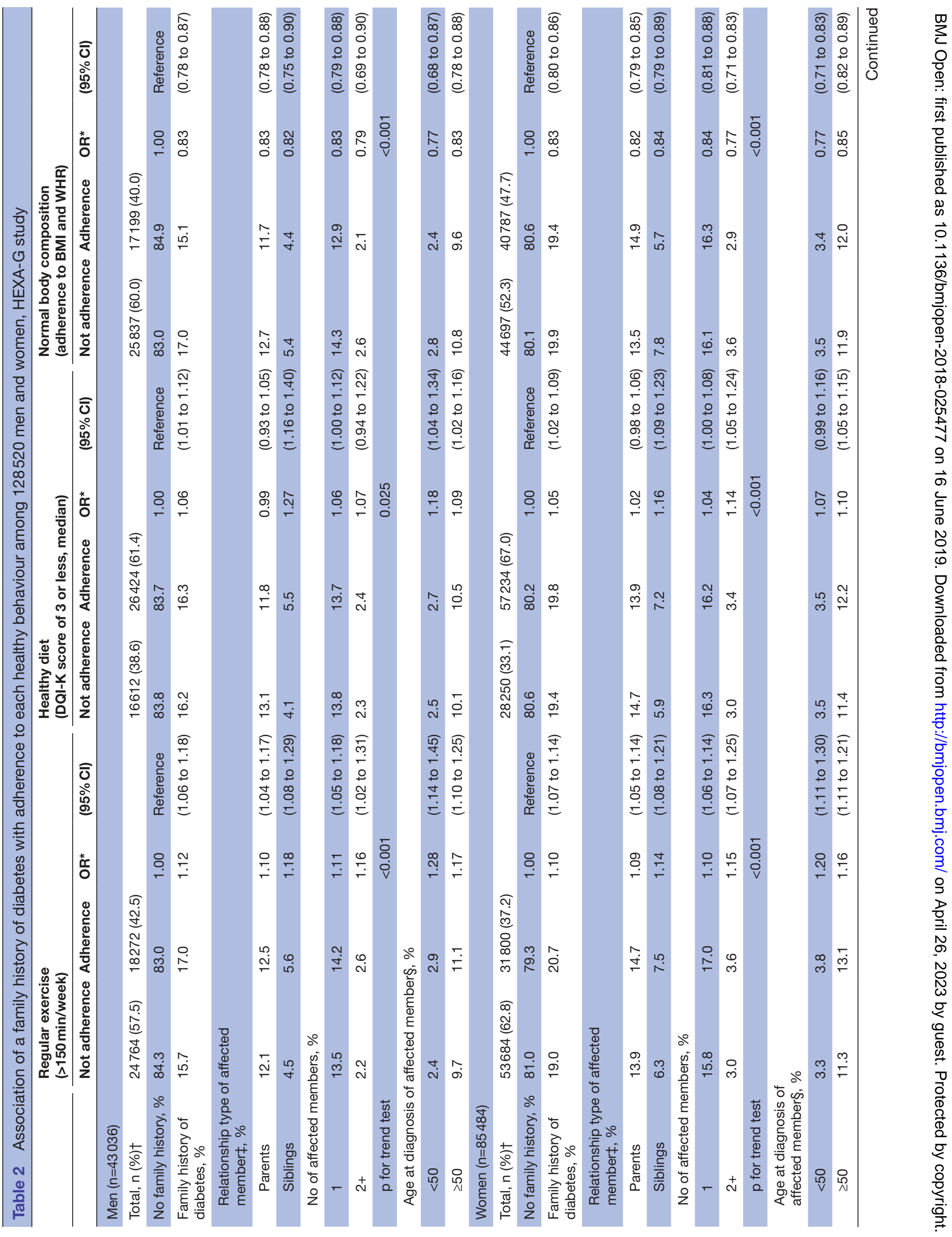




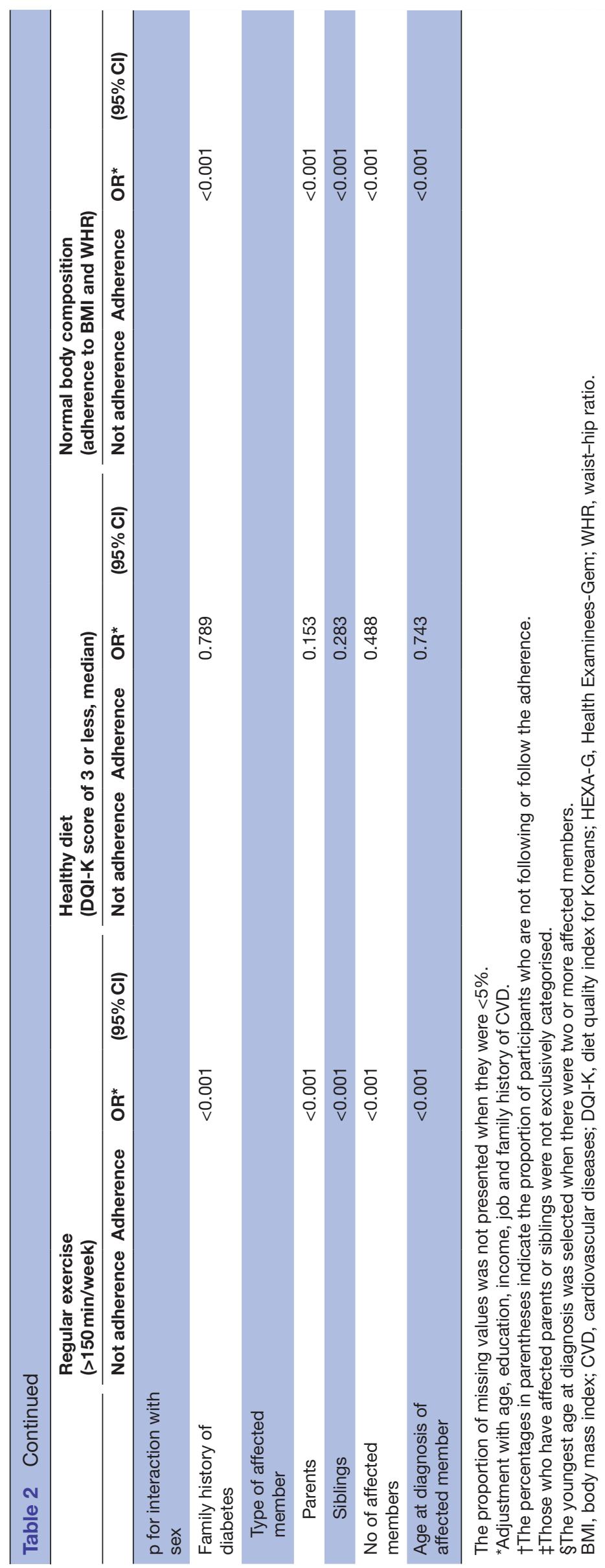

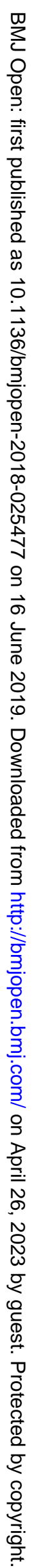


these associations were strengthened when the affected member was a sibling, the number of affected members was increased or the age at diagnosis of the affected member was younger than 50 years. The results of the trend test for the number of affected members were significant in all behaviours. The interactions between family history of diabetes and sex with adherence to regular exercise and having a normal body composition were significant, but the interactions with healthy diet were not. The results were not largely changed in the analysis of participants without a prior diagnosis of diabetes (online supplementary table 3), or diabetes, CVD and cancer (online supplementary table 4), or other diseases reported in the questionnaire, including hypertension, hyperlipidemia, gastrointestinal diseases, polyps, liver diseases, gallstones, asthma, bronchial diseases, allergy, thyroid disease, arthritis, osteoporosis, eye diseases and depression, along with diabetes, CVD and cancer (data not shown).

The two clusters found in men were defined as 'moderately active and healthy diet, but abnormal body composition' (60.0\% of men) and 'moderately active, healthy diet and normal body composition' (40.0\%), and the four clusters in women were defined as 'unhealthy behaviours' (11.7\% of women), 'healthy diet' (21.9\%) 'moderately active and normal body composition' (21.3\%) and 'healthy diet and balanced behaviour' (45.0\%) (figure 2). In men, only the proportion adhering to having a normal body composition was distinguished between the two clusters. Table 3 presents the associations between family history of diabetes and clusters of behaviours in men and women. In men, the moderately active and healthy diet, but abnormal body composition cluster was used as a reference group and those having a family history of diabetes were less likely to be in the moderately active, healthy diet and normal body composition cluster compared with moderately active and healthy diet, but abnormal body composition cluster (table 3), which was similar to the association of family history and adherence to having a normal body composition. In women, those having a family history of diabetes were less likely to adhere to the moderately active and normal body composition cluster, but those who had affected siblings were more likely to adhere to the healthy diet and balanced behaviours cluster. The magnitude and significance of the associations between family history-related characteristics and the healthy diet cluster were similar to the associations with healthy diet as an individual factor. In the rest of the clusters, the direction of the associations with family history was similar to those of adherence to having a normal body composition. The distribution of basic characteristics of the healthy clusters was also similar to the distribution of basic characteristics according to adherence to having a normal body composition, rather than regular exercise or a healthy diet (online supplementary tables 5 and 6 ).

\section{DISCUSSION}

The present study showed a positive association of a family history of diabetes with adherence to regular exercise and a healthy diet but a negative association with adherence to having a normal body composition. The magnitude of these associations was strengthened when the affected member was a sibling, the number of affected members increased or the age at diagnosis of the affected member was younger than 50 years. Further, adherence to normal body composition was an important component in determining the clusters of behaviours, especially in men; those with a family history of diabetes were less likely to adhere to a 'normal body composition' cluster.

Previous studies have reported inconsistent associations between a family history of diabetes and regular exercise. One study reported a positive association between a family history of diabetes and participation in physical activity, ${ }^{21}$ which is consistent with our results, whereas most previous results indicated non-significant associations with physical activity, ${ }^{22-26}$ except for one study showing a negative association. ${ }^{19}$ There were two reports that individuals who had family members with diabetes were more likely to have a healthy diet, as assessed by daily consumption of vegetables and low-fat milk and the use of vegetable margarine or no fat on bread ${ }^{20}$ or by having a lower caloric intake than those without a family history of diabetes, ${ }^{22}$ but non-significant associations were found in other studies. ${ }^{2325} 26$ For body composition, most previous results were consistent with our study, ${ }^{19-22} 2426$ with the exception of two studies showing a non-significant association. ${ }^{23} 25$ A possible reason for the non-significant associations between a family history of diabetes and adherence to healthy behaviours in previous studies, especially regarding regular exercise and a healthy diet, is that there was a lack of power to capture the effects of family history due to a relatively small sample size of $<11000$ participants and with a prevalence of a family history of diabetes at $\sim 20.0 \%$.

It was known that family history could induce the perception of disease risk. ${ }^{1516}$ Those with a family history of diabetes tried to reduce or control the familial risk by adopting certain lifestyles or behaviors ${ }^{40}$ because factors such as physical activity, dietary habits and obesity were perceived as risk factors for the disease. ${ }^{41}$ However, there were inverse associations between healthy behaviours, such as regular exercise or healthy diet, and normal body composition. As explained above, these associations were also found in previous studies, ${ }^{20}$ especially for obesity, as those with a family history of diabetes showed higher BMI or waist circumference in most studies. ${ }^{19-22} 2426$ The onset of disease in family members means that there had been intergenerational transmission of poor lifestyle behaviours, such as smoking, drinking alcohol, being overweight and being inactive that had been shared within the family before the onset of the disease. ${ }^{42}$ Among those behaviours, it has been known that obesity is highly influenced by genetic factors, and there is common genomic architecture in obesity and type 2 diabetes. ${ }^{43}$ 


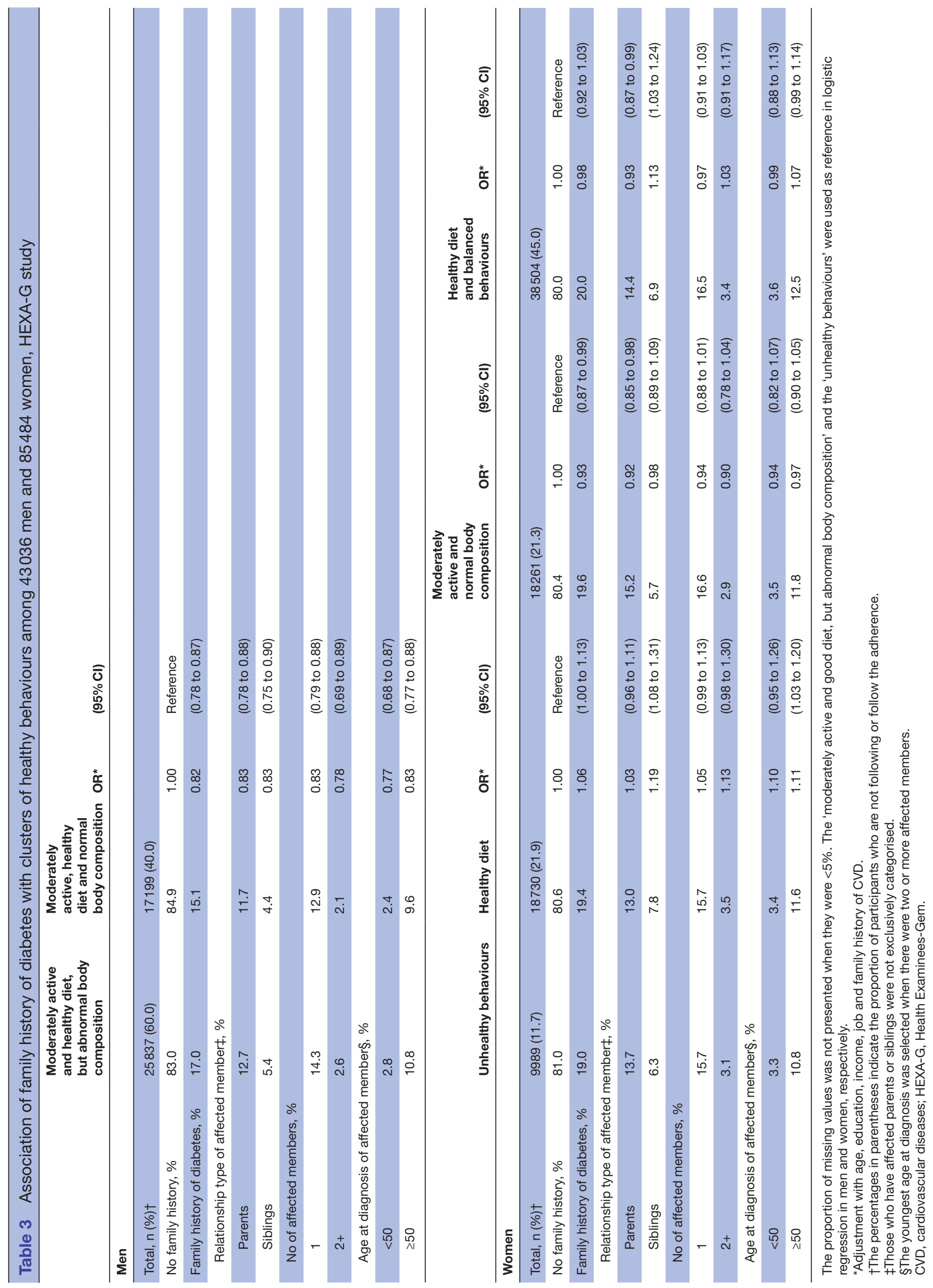


Those who have the common genetic architectures of obesity and type 2 diabetes are more likely to become obese, and the effects of regular exercise or healthy diet on obesity-related body composition can be attenuated. ${ }^{44}$ In addition to these genetic causes, barriers to healthy behaviours such as a lack of time or competing priorities typically perceived in middle-aged adults ${ }^{45}$ could make it difficult to maintain long-term and regular energy balance-related behaviours for achieving the ideal body composition.

The present study found that characteristics of family history, such as the relationship type, number and age at diagnosis of affected family members, had a slightly different effect on healthy behaviours. These results confirmed the previous reports that the characteristics of family history could influence an individual's perception of his or her vulnerability to diseases and could thus influence lifestyle behaviours. ${ }^{27}$ Regarding the relationship type of family member, environmental factors shared differently with parents and siblings might also influence adherence to healthy behaviours. ${ }^{47}$ Further study should confirm which environmental or social factors shared with family members caused the difference in association with behavioural factors when a family history occurred.

Behaviours typically co-occur and cluster together rather than being independent. For example, those with a lower BMI may participate in physical activity or have healthy dietary habits. ${ }^{48}$ Thus, information about which risk factors cluster together can help clinicians screen the vulnerable population to target health promotion strategies. ${ }^{49}$ To our knowledge, no previous studies considered the clusters of behaviours with family history. In the analysis of clustered groups, we found that there were broadly two types of clusters, with or without adherence to having a normal body composition by comparing the distribution of the basic characteristics or family history of diabetes according to adherence to normal body composition, as individual factors, with the cluster including normal body composition (online supplementary tables 5 and 6). Taken together, we could infer that the clusters of behaviours were mostly derived from having a normal body composition.

Although smoking and alcohol are known as major behavioural risk factors for death and disease worldwide ${ }^{50}$ they were not considered as behavioural factors in the present study because they were suggested only in the management guidelines for diabetes patients but not in the preventive guidelines by the American Diabetes Association, National Institute of Diabetes and Digestive and Kidney Disease, and the Korea Centers for Disease Control and Prevention. ${ }^{12-14}$ They were also not included in the list of covariates because we could not determine whether smoking and alcohol consumption were confounders, intermediates or colliders in the DAG. Although we could not define smoking or alcohol consumption as a confounder through the results of previous studies or the DAG, we also found that the results were almost unchanged when those variables were included in the confounder set of the model (data not shown).

The present study has some limitations. First, family history of diabetes was collected with a self-reported questionnaire. This can cause recall bias if the participant does not remember the exact diagnosis. However, test-retest reliability for family history of diabetes was evaluated by estimating Cohen's kappa coefficient and was outstanding at 0.90 in the repeatedly measured participants, with a median follow-up duration of 4.2 years after the baseline recruitment (data not shown). Second, the use of self-reported lifestyle behaviours may lead to over-ascertainment of healthy behaviours and could have biased estimates of the associations in the present study. Third, our study did not distinguish between family history of type 1 and type 2 diabetes. However, we ruled out the effect of type 1 diabetes by excluding participants with offspring diagnosed with diabetes because type 1 diabetes mostly develops during childhood.

\section{CONCLUSION}

We found that participants with a diabetes-affected family member were living more actively and had healthier dietary habits but were less likely to have a normal body composition, which is a key factor for determining behavioural patterns. The results of the current study could be used to develop a manageable intervention focusing on reducing diabetes risk by helping high-risk populations, such as those with affected family members, to successfully achieve a normal body composition. Health improvement policy and campaigns targeting integrated health behaviours will be needed to reduce the burden of diseases and improve public health.

\section{Author affiliations}

${ }^{1}$ Department of Biomedical Sciences, Seoul National University Graduate School, Seoul, Korea

${ }^{2}$ Department of Preventive Medicine, Seoul National University College of Medicine, Seoul, Korea

${ }^{3}$ Cancer Research Institute, Seoul National University, Seoul, Korea

${ }^{4}$ Department of Preventive Medicine, Kangwon National University School of Medicine, Kangwon, Korea

${ }^{5}$ Department of Environmental Health, College of Natural Science, Korea National Open University, Seoul, Korea

${ }^{6}$ JW Lee Center for Global Medicine, Seoul National University College of Medicine, Seoul, Korea

${ }^{7}$ Division of Cancer Epidemiology and Genetics, Department of Health and Human Services, National Cancer Institute, National Institutes of Health, Bethesda,

Maryland, USA

${ }^{8}$ Department of Family Medicine, Seoul National University College of Medicine, Seoul, Korea

${ }^{9}$ Institute of Environmental Medicine, Seoul National University Medical Research Center, Seoul, Korea

Contributors $\mathrm{JC}$ and $\mathrm{J}-\mathrm{YC}$ contributed to the conception and design of the study, acquisition of the data and the analysis and interpretation of the data. S-AL, $\mathrm{K}-\mathrm{ML}, \mathrm{AS}, \mathrm{JO}$, JYP, MS, JJY, J-kL and DK contributed to acquisition of data and interpretation of data. JC and JYC contributed to drafting the article and revising it after reviewing. S-AL, K-ML, AS, JO, JYP, MS, JJY, J-kL and DK reviewing it. All authors approved the final draft. 
Funding This work was supported by the research programme funded by the Korea Centers for Disease Control and Prevention (2004-E71004-00, 2005E71011-00, 2005-E71009-00, 2006-E71001-00, 2006-E71004-00, 2006-E7101000, 2006-E71003-00, 2007-E71004-00, 2007-E71006-00, 2008-E71006-00, 2008-E71008-00, 2009-E71009-00, 2010-E71006-00, 2011-E71006-00, 2012E71001-00 and 2013-E71009-00), Seoul National University Hospital (2019) and Ministry of Education of the Republic of Korea and the National Research Foundation of Korea (NRF-2018R1A2A3075397).

Competing interests None declared.

Patient consent for publication Obtained.

Ethics approval The Institutional Review Board of Seoul National University Hospital, Seoul, Korea approved the study (IRB No. 0608-018-179).

Provenance and peer review Not commissioned; externally peer reviewed.

Data sharing statement The data that support the findings of this study are available from Korea Center for Disease Control and Prevention but restrictions apply to the availability of these data, which were used under license for the current study, and so are not publicly available. Data are, however, available from the authors upon reasonable request and with permission of Korea Center for Disease Control and Prevention.

Open access This is an open access article distributed in accordance with the Creative Commons Attribution Non Commercial (CC BY-NC 4.0) license, which permits others to distribute, remix, adapt, build upon this work non-commercially, and license their derivative works on different terms, provided the original work is properly cited, appropriate credit is given, any changes made indicated, and the use is non-commercial. See: http://creativecommons.org/licenses/by-nc/4.0/.

\section{REFERENCES}

1. NCD Risk Factor Collaboration. Worldwide trends in diabetes since 1980: a pooled analysis of 751 population-based studies with $4 \cdot 4$ million participants. The Lancet 2016;387:1513-30.

2. Wang $H$, Naghavi $M$, Allen $C$, et al. Global, regional, and national life expectancy, all-cause mortality, and cause-specific mortality for 249 causes of death, 1980-2015: a systematic analysis for the Global Burden of Disease Study 2015. The Lancet 2016;388:1459-544.

3. Vos T, Allen C, Arora M, et al. Global, regional, and national incidence, prevalence, and years lived with disability for 310 diseases and injuries, 1990-2015: a systematic analysis for the Global Burden of Disease Study 2015. Lancet 2016;388:1545-602.

4. Korean Diabetes Association. Diabetes Fact Sheet in Korea 2016. 2017 http://www.diabetes.or.kr/pro/news/admin.php?category=A\& code=admin\&number=1428\&mode=view (accessed 13 Feb 2018).

5. Koo BK, Kim SW, Yi KH, et al. Changing relative contribution of abdominal obesity and a family history of diabetes on prevalence of diabetes mellitus in Korean men and women aged 30-49 years from 2001 to 2010. J Diabetes 2015;7:465-72

6. Katulanda P, Ranasinghe P, Jayawardena R, et al. The influence of family history of diabetes on disease prevalence and associated metabolic risk factors among Sri Lankan adults. Diabet Med 2015;32:314-23.

7. Knowler WC, Barrett-Connor E, Fowler SE, et al. Reduction in the incidence of type 2 diabetes with lifestyle intervention or metformin. N Engl J Med 2002;346:393-403.

8. Li G, Zhang P, Wang J, et al. The long-term effect of lifestyle interventions to prevent diabetes in the China Da Qing Diabetes Prevention Study: a 20-year follow-up study. The Lancet 2008;371:1783-9.

9. Lv J, Yu C, Guo Y, et al. Adherence to a healthy lifestyle and the risk of type 2 diabetes in Chinese adults. Int $J$ Epidemiol 2017;46:1410-20.

10. Hariri S, Yoon PW, Qureshi N, et al. Family history of type 2 diabetes: a population-based screening tool for prevention? Genet Med 2006;8:102-8.

11. Harrison TA, Hindorff LA, Kim H, et al. Family history of diabetes as a potential public health tool. Am J Prev Med 2003;24:152-9.

12. Standards of Medical Care in Diabetes-2017: Summary of Revisions. Diabetes Care 2017;40(Suppl 1):S4-S5

13. National Institute of Diabetes and Digestive and Kidney Disease. Preventing Type 2 Diabetes. 2016 https://www.niddk.nih.gov/healthinformation/diabetes/overview/preventing-type-2-diabetes (accessed 7 Jan 2018).

14. Korea Centers for Disease Control \& Prevention. Previnting the diabetes. 2016 http://cdc.go.kr/CDC/contents/CdcKrContentView.
jsp?cid=26646\&menulds=HOME001-MNU1130-MNU0754MNU1098-MNU1455 (accessed 8 Jan 2018).

15. Ferrer R, Klein WM. Risk perceptions and health behavior. Curr Opin Psychol 2015;5:85-9.

16. Darlow S, Goodman MS, Stafford JD, et al. Weight perceptions and perceived risk for diabetes and heart disease among overweight and obese women, Suffolk County, New York, 2008. Prev Chronic Dis 2012;9:E81

17. Acheson LS, Wang C, Zyzanski SJ, et al. Family history and perceptions about risk and prevention for chronic diseases in primary care: a report from the family healthware impact trial. Genet Med 2010;12:212-8.

18. Imes CC, Lewis FM. Family history of cardiovascular disease, perceived cardiovascular disease risk, and health-related behavior: a review of the literature. J Cardiovasc Nurs 2014;29:108-29.

19. Hilding A, Eriksson AK, Agardh EE, et al. The impact of family history of diabetes and lifestyle factors on abnormal glucose regulation in middle-aged Swedish men and women. Diabetologia 2006;49:2589-98.

20. Isomaa B, Forsén B, Lahti K, et al. A family history of diabetes is associated with reduced physical fitness in the Prevalence, Prediction and Prevention of Diabetes (PPP)-Botnia study. Diabetologia 2010;53:1709-13.

21. Goodrich KM, Crowley SK, Lee DC, et al. Associations of cardiorespiratory fitness and parental history of diabetes with risk of type 2 diabetes. Diabetes Res Clin Pract 2012;95:425-31.

22. Scott RA, Langenberg C, Sharp SJ, et al. The link between family history and risk of type 2 diabetes is not explained by anthropometric, lifestyle or genetic risk factors: the EPIC-InterAct study. Diabetologia 2013;56:60-9.

23. Zlot Al, Cox SL, Silvey K, et al. The effect of chronic disease family history on healthcare provider practice and patient behavior among Oregonians. Public Health Genomics 2012;15(3-4):189-200.

24. Ning F, Pang Z, Laatikainen $T$, et al. Joint effect of family history of diabetes with obesity on prevalence of type 2 diabetes mellitus among Chinese and Finnish men and women. Can J Diabetes 2013;37:65-71.

25. Sakurai M, Nakamura K, Miura K, et al. Family history of diabetes, lifestyle factors, and the 7-year incident risk of type 2 diabetes mellitus in middle-aged Japanese men and women. $J$ Diabetes Investig 2013:4:261-8.

26. Akhuemonkhan E, Lazo M. Association between family history of diabetes and cardiovascular disease and lifestyle risk factors in the United States population: The 2009-2012 National Health and Nutrition Examination Survey. Prev Med 2017;96:129-34.

27. Yoon PW, Scheuner MT, Jorgensen C, et al. Developing Family Healthware, a family history screening tool to prevent common chronic diseases. Prev Chronic Dis 2009;6:A33.

28. McAloney K, Graham H, Law C, et al. A scoping review of statistical approaches to the analysis of multiple health-related behaviours. Prev Med 2013:56:365-71.

29. Poortinga $W$. The prevalence and clustering of four major lifestyle risk factors in an English adult population. Prev Med 2007;44:124-8.

30. Prochaska JJ, Prochaska JO. A Review of Multiple Health Behavio Change Interventions for Primary Prevention. Am J Lifestyle Med 2011;5:208-21.

31. Health Examinees Study Group. The Health Examinees (HEXA) study: rationale, study design and baseline characteristics. Asian Pac $J$ Cancer Prev 2015;16:1591-7.

32. Kim Y, Han BG. Cohort Profile: The Korean Genome and Epidemiology Study (KoGES) Consortium. International journal of epidemiology 2016 (published Online First: 2016/04/17).

33. Lascar N, Brown J, Pattison H, et al. Type 2 diabetes in adolescents and young adults. Lancet Diabetes Endocrinol 2018;6:69-80.

34. WHO Guidelines Approved by the Guidelines Review Committee. Global Recommendations on Physical Activity for Health. 2010. Geneva: World Health Organization Copyright (c) World Health Organization, 2010.

35. Lim J, Lee Y, Shin S, et al. An association between diet quality index for Koreans (DQI-K) and total mortality in Health Examinees Gem (HEXA-G) study. Nutr Res Pract 2018;12:258.

36. World Health Organization. Global database on Body Mass Index BMI Classification. 2006. 16: World Health Organization, 2016. Retrieved.

37. World Health Organization. Waist circumference and waist-hip ratio: Report of a WHO expert consultation, Geneva, 8-11, 2008.

38. Textor J, Hardt J, Knüppel S. DAGitty: a graphical tool for analyzing causal diagrams. Epidemiology 2011;22:745.

39. Dean D. Wexler J: What's New in SAS Enterprise Miner 13.1 Proceedings of the SAS Global Forum 2014 Conference. Washington, DC, 2014. Citeseer. 
40. Walter FM, Emery J. Perceptions of family history across common diseases: a qualitative study in primary care. Fam Pract 2006;23:472-80.

41. Skøt L, Nielsen JB, Leppin A. Who perceives a higher personal risk of developing type 2 diabetes? A cross-sectional study on associations between personality traits, health-related behaviours and perceptions of susceptibility among university students in Denmark. BMC Public Health 2018;18:972.

42. Wickrama KA, Conger RD, Wallace LE, et al. The intergenerational transmission of health-risk behaviors: adolescent lifestyles and gender moderating effects. J Health Soc Behav 1999;40:258-72.

43. Grarup N, Sandholt $\mathrm{CH}$, Hansen T, et al. Genetic susceptibility to type 2 diabetes and obesity: from genome-wide association studies to rare variants and beyond. Diabetologia 2014;57:1528-41.

44. Graff M, Scott RA, Justice AE, et al. Genome-wide physical activity interactions in adiposity - A meta-analysis of 200,452 adults. PLoS Genet 2017;13:e1006528.

45. Lee C, Ory MG, Yoon J, et al. Neighborhood walking among overweight and obese adults: age variations in barriers and motivators. J Community Health 2013;38:12-22.
46. Seguin R, Connor L, Nelson M, et al. Understanding barriers and facilitators to healthy eating and active living in rural communities. $J$ Nutr Metab 2014;2014:1-8.

47. Muñoz M, Pong-Wong R, Canela-Xandri O, et al. Evaluating the contribution of genetics and familial shared environment to common disease using the UK Biobank. Nat Genet 2016;48:980-3.

48. Besson $\mathrm{H}$, Ekelund $\mathrm{U}$, Luan $\mathrm{J}$, et al. A cross-sectional analysis of physical activity and obesity indicators in European participants of the EPIC-PANACEA study. Int J Obes 2009;33:497-506.

49. Charreire H, Casey R, Salze P, et al. Leisure-time physical activity and sedentary behavior clusters and their associations with overweight in middle-aged French adults. Int $J$ Obes 2010;34:1293-301.

50. Forouzanfar MH, Alexander L, Anderson HR, et al. Global, regional, and national comparative risk assessment of 79 behavioural, environmental and occupational, and metabolic risks or clusters of risks in 188 countries, 1990-2013: a systematic analysis for the Global Burden of Disease Study 2013. The Lancet 2015;386:2287-323. 